\title{
A euglycaemic/non-diabetic perinatal environment does not alleviate early beta cell maldevelopment and type 2 diabetes risk in the GK/Par rat model
}

\author{
A. Chavey • D. Bailbé • L. Maulny • J. P. Renard • \\ J. Movassat • B. Portha
}

Received: 1 March 2012 /Accepted: 30 July 2012 /Published online: 12 October 2012

(C) Springer-Verlag Berlin Heidelberg 2012

\begin{abstract}
Aims/hypothesis We used the GK/Par rat, a spontaneous model of type 2 diabetes with early defective beta cell neogenesis, to determine whether the development of GK/Par offspring in a non-diabetic intrauterine/postnatal environment would prevent the alteration of fetal beta cell mass $(\mathrm{BCM})$ and ultimately decrease the risk of diabetes later in adult life.

Methods We used an embryo-transfer approach, with fertilised GK/Par ovocytes (oGK) being transferred into pregnant Wistar (W) or GK/Par females (pW and pGK). Offspring were phenotyped at fetal age E18.5 and at 10 weeks of age, for BCM, expression of genes of pancreatic progenitor cell regulators (Igf2, $\operatorname{Ig} f 1 r$, Sox $9, P d x I$ and $N g n 3)$, glucose tolerance and insulin secretion.

Results (1) Alterations in neogenesis markers/regulators and $\mathrm{BCM}$ were as severe in the oGK/pW E18.5 fetuses as in the oGK/pGK group. (2) Adult offspring from oGK transfers into GK (oGK/pGK/sGK) had the expected diabetic phenotype compared with unmanipulated GK rats. (3) Adult offspring from oGK reared in $\mathrm{pW}$ mothers and milked by GK
\end{abstract}

Electronic supplementary material The online version of this article (doi:10.1007/s00125-012-2733-8) contains peer-reviewed but unedited supplementary material, which is available to authorised users.

A. Chavey $\cdot$ D. Bailbé $\cdot$ J. Movassat $\cdot$ B. Portha $(\bowtie)$

Université Paris-Diderot, Sorbonne Paris Cité, Laboratoire B2PE,

Unité BFA, CNRS EAC 4413,

Bâtiment Buffon - 5ème étage - pièce 552A,

4, Rue Lagroua Weill Hallé, Case 7126,

75205 Paris Cedex13, France

e-mail: portha@univ-paris-diderot.fr

L. Maulny $\cdot$ J. P. Renard

INRA UMR 1198 ,

Jouy, France foster mothers had reduced BCM, basal hyperglycaemia, glucose intolerance and low insulin, to an extent similar to that of oGK/pGK/sGK offspring. (4) In adult offspring from oGK transferred into $\mathrm{pW}$ mothers and milked by their $\mathrm{W}$ mothers $(\mathrm{oGK} / \mathrm{pW} / \mathrm{sW})$, the phenotype was similar to that in $\mathrm{oGK} / \mathrm{pGK} / \mathrm{sGK}$ or oGK/pW/sGK offspring.

Conclusions/interpretation These data support the conclusion that early $\mathrm{BCM}$ alteration and subsequent diabetes risk in the GK/Par model are not removed despite normalisation of the prenatal and postnatal environments, either isolated or combined.

Keywords Beta cell mass - Diabetes risk - Embryo transfer . GK/Par rat $\cdot \operatorname{Igflr} \cdot \operatorname{Igf2} \cdot$ Maternal diabetes $\cdot \operatorname{Ngn} 3 \cdot P d x 1$. Sox 9
Abbreviations
ACM
$\mathrm{BCM}$
E13.5
E18.5
IGF1R
IPGTT
$\mathrm{nGK} / \mathrm{sGK}$
Total pancreatic alpha cell mass
Total pancreatic beta cell mass
Embryonic age 13.5
Embryonic age 18.5
IGF1 receptor
Intraperitoneal glucose tolerance test
nGK/sW Pups from GK/Par mothers and suckled
Pups from GK/Par mothers and suckled by GK/Par foster mothers by $\mathrm{W}$ foster mothers
NGN3 Neurogenin 3
$\mathrm{nW} / \mathrm{sW} \quad$ Pups from W mothers and suckled by W foster mothers
oGK/pGK Fetuses from GK/Par ovocyte transfer into GK/Par females
oGK/pGK/sGK Adults from GK/Par ovocyte transfer into GK/Par females and suckled by GK/Par mothers until young adult age 


\begin{tabular}{|c|c|}
\hline $\mathrm{oGK} / \mathrm{pW}$ & $\begin{array}{l}\text { Fetuses from GK/Par ovocyte transfer } \\
\text { into } \mathrm{W} \text { females }\end{array}$ \\
\hline $\mathrm{oGK} / \mathrm{pW} / \mathrm{sGK}$ & $\begin{array}{l}\text { Adults from GK/Par ovocyte transfer } \\
\text { into W females and suckled by GK/Par } \\
\text { foster mothers until } 4 \text { weeks of age }\end{array}$ \\
\hline $\mathrm{oGK} / \mathrm{pW} / \mathrm{sW}$ & $\begin{array}{l}\text { Adults from GK/Par ovocyte transfer } \\
\text { into W females and suckled by } \\
\text { W mothers until } 4 \text { weeks of age }\end{array}$ \\
\hline $\mathrm{oW} / \mathrm{pW}$ & $\begin{array}{l}\text { Fetuses from } \mathrm{W} \text { ovocyte transfer into } \\
\mathrm{W} \text { females }\end{array}$ \\
\hline PDX1 & Pancreatic and duodenal homeobox 1 \\
\hline QTL & Quantitative trait locus \\
\hline SOX9 & SRY (sex-determining region Y) box 9 \\
\hline
\end{tabular}

\section{Introduction}

A role for maternal transmission of type 2 diabetes was first suggested by epidemiological studies [1-6]. Fetal exposure to type 2 diabetes as an environmental factor that may explain the maternal transmission of type 2 diabetes was first demonstrated in Pima Indians, a population with the highest prevalence of type 2 diabetes reported in the world [7]. In an attempt to negate the confounding effect of genetic factors related to type 2 diabetes, the effect of in utero exposure to type 1 diabetes was studied in a group of adult offspring free from immunological markers of type 1 diabetes: a $33 \%$ prevalence of impaired glucose tolerance was reported in offspring of mothers compared with none of the offspring of fathers [8]. Taken together, these findings suggest that in utero exposure to diabetes is associated with abnormal glucose homeostasis in offspring of diabetic mothers and may be involved in the excess maternal transmission in type 2 diabetes.

Since maternal genes normally always develop in the same uterus, studies to dissect the relative contributions of genes and intrauterine diabetic environment have proved difficult to design. Experimental studies that have attempted to address this important issue directly are scarce. It is hard to maintain euglycaemia in pregnant diabetic rats, and it has not been possible to study the effects of rearing diabetesprone fetuses in their maternal uterus while avoiding maternal diabetes. Any relevant model suitable for dissociating the impact of genetic predisposition of the progeny from that of the diabetic maternal environment would enter the pregnancy in a euglycaemic state, become exposed to normoglycaemia during the pregnancy, and return postpartum to a normoglycaemic environment. It would also allow the study of long-term phenotype consequences of genetic predisposition to diabetes independently of any maternal influence. The embryo-transfer paradigm between non-diabetic rats and diabetic rats proposed by Gill-Randall et al [9] offers an experimental strategy for addressing this question.
The aim of this study was to dissect in the GK rat, a relevant model of spontaneous type 2 diabetes, the respective roles of genetic and environmental factors contributing to the early impairment of total pancreatic beta cell mass (BCM) development and the subsequent onset of diabetes [10-12]. It relies on the use of embryo-transfer experiments to investigate the influence of a euglycaemic/non-diabetic perinatal diabetic environment as found in a Wistar mother on the extent of BCM insufficiency (fetal stage and adult age) in a diabetes-prone GK/Par conceptus (in the presence of diabetes-predisposing genes). We also evaluated the risk of diabetes in adult GK/Par offspring raised under these conditions.

\section{Methods}

Animals All animal experimentation was conducted on GK/ Par rats (GK) and non-diabetic Wistar (W) rats from our local colonies maintained at the University Paris-Diderot animal core, and in accordance with accepted standards of animal care laid out in the French National Center for Scientific Research guidelines. The characteristics of the adult GK rats have been described previously [11].

Embryo harvesting and transfer procedures Embryos of the required genetic background ( $\mathrm{GK}$ or $\mathrm{W}$ ) were obtained from donor female rats caged in groups of up to four with a male of proven fertility (E0) and allowed to mate overnight. The following morning, coitus was confirmed by the presence of a vaginal plug, and embryos were harvested in the early afternoon at E0.5 (one-cell stage). All embryos were recovered and transplanted surgically into recipient females as described by Cozzi et al [13].

Experimental groups Eighteen experimental groups were designed. Ovocytes obtained from GK rats (oGK) were transferred back into pseudo-pregnant GK female rats (pGK) (oGK/pGK group). This group was compared with a non-manipulated GK stock group (GK stock). Ovocytes obtained from $\mathrm{W}$ rats $(\mathrm{oW})$ were transferred back into pseudo-pregnant $\mathrm{W}$ female rats $(\mathrm{pW})(\mathrm{oW} / \mathrm{pW})$. This was our control non-diabetic group. This group was compared with a non-manipulated W stock group. Ovocytes obtained from GK rats were transferred into pseudo-pregnant $\mathrm{W}$ females $(\mathrm{oGK} / \mathrm{pW})$ in order to study the effects of rearing GK embryos in a euglycaemic uterus. GK newborns (nGK) from GK mothers were suckled by W foster mothers (nGK/ $\mathrm{sW}$ ) (see electronic supplementary material [ESM] Methods for additional details). This group was compared with nonmanipulated $\mathrm{W}$ stock newborns $(\mathrm{nW} / \mathrm{sW})$ and nonmanipulated GK stock newborns $(\mathrm{nGK} / \mathrm{sGK})$ raised in parallel. 
Accordingly, the following groups of rats were studied: oGK/pGK E13.5 fetuses, oGK/pW E13.5 fetuses, oW/pW E13.5 fetuses, GK stock E13.5 fetuses, W stock E13.5 fetuses, oGK/pGK E18.5 fetuses, oGK/pW E18.5 fetuses, oW/pW E18.5 fetuses, GK stock E18.5 fetuses, W stock E18.5 fetuses, oGK/pGK/sGK 10-week-old adults, oGK/pW/sW 10-weekold adults, oGK/pW/sGK 10-week-old adults, $\mathrm{nGK} / \mathrm{sGK}$ rats (4- and 8-week-old), nGK/sW rats (4- and 8-week-old), $\mathrm{nW} / \mathrm{sW}$ rats (4- and 8-week-old), GK/Par stock 10-week-old adults (10 weeks), W stock 10-week-old adults.

Fetal samples On day 13.5 (E13.5) or day 18.5 of gestation (E18.5), pregnant rats of oW/pW, oGK/pGK, oGK $/ \mathrm{pW}$, $\mathrm{W}$ stock or GK stock were injected i.p. with pentobarbital sodium ( $1 \mathrm{ml} / \mathrm{kg}$ body weight), and their fetuses and placentas were extracted and weighed. In E18.5 fetuses only, blood samples were obtained from axillary vessels. Their pancreases were then excised and weighed. For western blot analysis, pancreases were immersed in liquid nitrogen and stored at $-70^{\circ} \mathrm{C}$ until analysis. For immunohistochemistry and morphometric analysis, six to seven E18.5 pancreases in each group (from at least three different litters) were fixed in aqueous Bouin solution, embedded in paraplast, serially sectioned $(6 \mu \mathrm{m})$ and mounted on slides.

Glucose tolerance and insulin secretion tests Intraperitoneal glucose tolerance tests (IPGTTs; $1 \mathrm{~g}$ glucose $/ \mathrm{kg}$ body weight) were performed in the 4-week- and 8-week-old nonanaesthetised rats, as previously described [14]. Animals from at least three different litters were used in each group. Blood samples were collected from the tail vein before and 15, 30 and $60 \mathrm{~min}$ after glucose administration. IVGTTs $(0.5 \mathrm{~g}$ glucose $/ \mathrm{kg}$ body weight) were performed under pentobarbital sodium anaesthesia $(80 \mu \mathrm{l} / 100 \mathrm{~g}$ body weight i.p.; Ceva Santé Animal, Libourne, France) in the 10-week-old rats, as previously described [15]. Blood samples were collected sequentially from the tail vein before and 5, 10, 15, 20,30 and $60 \mathrm{~min}$ after the injection of glucose. Plasma glucose and insulin were determined at each time point, as previously described [14]. At the end of the IVGTT, pancreases were excised and weighed. For determination of insulin content, pancreases were homogenised and centrifuged $(1500 \mathrm{~g})$ at $4^{\circ} \mathrm{C}$ in an acid/alcohol solution (75\% ethanol, $1.5 \% 32.5 \mathrm{~mol} / 1 \mathrm{HCl}$ and $23.5 \%$ distilled water). The supernatant fraction was stored at $-20^{\circ} \mathrm{C}$ until insulin was assayed. For endocrine cell immunohistochemistry and morphometry, pancreases were fixed in aqueous Bouin solution and embedded in paraplast according to standard procedures.

Western blot analysis Gel electrophoresis and immunoblotting for the detection of IGF2 and IGF1 receptor (IGF1R) were performed as previously described [16] and detailed in ESM Methods.
Immunohistochemical analysis Immunostaining for insulin, glucagon and pancreatic and duodenal homeobox 1 (PDX1) were performed as previously described [15]. Details of the immunostaining procedures for neurogenin $3(\mathrm{NGN} 3)$ and SRY (sex-determining region Y) box 9 (SOX9) are provided in ESM Methods.

Morphometric image analysis Quantitative evaluation of pancreatic cell areas was performed in pancreatic sections using a computer-assisted image analysis system (Histolab 5.2 software; Microvision Instruments, Evry, France). Measurement of relative beta cell and alpha cell areas in the pancreas and calculation of BCM and alpha cell mass (ACM) were performed as previously described [14]. At least six sections were analysed for each pancreas. Eight (adult groups) or seven (fetal groups) rats were analysed per experimental group.

Calculations The insulin and glucose responses during the IPGTT or IVGTT were calculated as the incremental plasma insulin values integrated over a period of $60 \mathrm{~min}$ after the injection of glucose $\left(\Delta \mathrm{I}, \mathrm{pmol}^{-1} \mathrm{~min}^{-1}\right)$ and the corresponding increase in glucose concentration $\left(\Delta \mathrm{G}, \mathrm{mmoll}^{-1} \mathrm{~min}^{-1}\right)$, respectively. The insulinogenic index $(\Delta \mathrm{I} / \Delta \mathrm{G})$ represents the ratio of these two variables. All results are expressed as mean \pm SEM, with the number of observations and significance between group differences evaluated by ANOVA followed by Fisher's exact test (StatView 5.0; SAS Institute, Cary, NC, USA). A $p$ value $<0.05$ was considered significant.

\section{Results}

Fetal phenotype of offspring from GK/Par into GK/Par ovocyte transfers At E13.5, the mean body weight of oGK-transferred GK females $(249 \pm 6 \mathrm{~g}, n=7)$ and GK stock females $(246 \pm 4 \mathrm{~g}, n=12)$ was similar. It remained significantly lower $(p<0.05)$ in the oGK-transferred GK pregnant group than in the oW-transferred W pregnant group (279 \pm $10 \mathrm{~g}, n=7)$. The pattern of basal plasma glucose concentrations according to gestational age was similar in oGKtransferred GK females $(6.9 \pm 0.3 \mathrm{mmol} / \mathrm{l}, n=7)$ and GK stock females $(7.7 \pm 0.4 \mathrm{mmol} / \mathrm{l}, n=12)$. They always exhibited a higher plasma glucose level throughout the gestation period $(p<0.001)$ compared with oW-transferred W controls $(4.9 \pm 0.3 \mathrm{mmol} / \mathrm{l}, n=7)$.

We studied GK fetuses from GK ovocyte transfer into GK females. Comparison was made with age-matched fetuses from the GK stock and the oW/pW groups. At E13.5, the number of viable fetuses per litter was similar in the oGK/pGK ( $47 \pm 7 \%$ viability, seven litters) and oW/gW (53 $\pm 7 \%$ viability, 14 litters) groups. Mean placental weights were similar in the oGK/pGK $(250 \pm 8 \mathrm{mg}, n=7)$ and oW/pW $(243 \pm 5 \mathrm{mg}, n=12)$ groups. Mean body weight was 
significantly lower $(p<0.05)$ in the oGK/pGK fetuses $(74 \pm 6 \mathrm{mg}, n=7)$ than in the oW/pW fetuses $(96 \pm 6 \mathrm{mg}$, $n=7$ ). It did not differ from that in the GK stock fetal group (71 $\pm 2 \mathrm{mg}, n=12$ ).

At E18.5, the number of viable fetuses per litter was significantly decreased $(p<0.01)$ in the oGK/pGK compared with the oW/pW group, as was their mean body weight ( $p<$ 0.02 ) (Table 1). This indicates that oGK/pGK fetuses were growth-retarded compared with control oW/gW fetuses. oGK/ pGK fetuses, similar to GK stock fetuses, exhibited a higher plasma glucose concentration $(p<0.01)$ than $\mathrm{oW} / \mathrm{pW}$ controls. Their pancreatic weights were not significantly different, but their BCMs were 4.2-fold decreased $(p<0.001)$. Their BCMs were in fact slightly lower than those in the age-related GK stock fetuses, but the difference did not reach statistical significance. oGK/gGK ACM was not significantly altered from $\mathrm{oW} / \mathrm{gW}$ or GK stock values (Fig. 1 and Table 1).

During normal development, endocrine and exocrine cells of the pancreas are derived from multipotent progenitor cells expressing the transcription factors, PDX1 and SOX9 [17]. Furthermore, all five endocrine cell types including insulin ${ }^{+}$beta cells arise from a common transient endocrine progenitor marked by production of the transcription factor, NGN3. Here, we show for the first time that GK stock fetuses exhibit a $55 \%$ reduction in the number of $\mathrm{SOX}^{+}$ pancreatic progenitor cells at E18.5, together with a decreased number of $\mathrm{PDX}^{+} /$insulin ${ }^{-}$cells and $\mathrm{NGN}^{+}$cells (respectively, by $58 \%$ and $61 \%$ ), compared with control $\mathrm{oW} / \mathrm{pW}$ fetuses. Corresponding values in the oGK/pGK fetuses were not significantly different from those of GK stock fetuses (Fig. 2 and Table 1). At E18.5, oGK/pGK fetuses, similarly to GK stock fetuses, showed significantly $(p<0.05)$ decreased intrapancreatic accumulation of IGF2 and IGF1R proteins (decreased by $58 \%$ and $30 \%$, respectively) compared with control oW/pW values at the same age (Fig. 1 and Table 1). These data confirm and extend our earlier reports of decreased transcript levels of $P d x 1$, Neurog 3 (also known as $N g n 3$ ) and $\operatorname{Ig} 2[16,18]$ and reduction in pancreatic IGF2 and IGF1R protein levels [16] in E18.5 GK stock fetuses.

Adult offspring from GK into GK ovocyte transfers (oGK/ $p G K / s G K)$ have the expected diabetic phenotype To assess any possible effect of ovocyte transfer on long-term glucose homeostasis and BCM in adults, we studied offspring from nine litters from GK ovocyte transfer into GK females and suckled by GK mothers (oGK/pGK/sGK group) until a young adult age. From the data obtained (Figs 3 and 4, Table 2, ESM Results), one can conclude that there is no evidence that the experimental GK ovocyte transfer procedure itself modified the risk of diabetes in the GK offspring from either sex, despite distinct aggravation of some pancreatic beta cell indicators.
Fetal phenotype of offspring from GK into W ovocyte transfers $(o G K / p W)$ On day 13.5 of gestation, mean body weight and basal plasma glucose concentrations were similar in oGK-transferred W females (respectively, $269 \pm 8 \mathrm{~g}$ and $4.6 \pm 0.1 \mathrm{mmol} / 1, n=12$,) and oW-transferred $\mathrm{W}$ females (respectively, $279 \pm 10 \mathrm{~g}$ and $4.9 \pm 0.3 \mathrm{mmol} / 1, n=7$ ). W females receiving GK ovocytes always exhibited a normal plasma glucose level throughout the gestation period, in contrast with the hyperglycaemic GK mothers (Table 1).

GK fetuses from GK ovocyte transfer into $\mathrm{W}$ females were compared with age-related fetuses from the oGK/pGK and $\mathrm{oW} / \mathrm{gW}$ groups. At E13.5 as well as E18.5, the number of viable fetuses per litter and placental weights were similar in the $\mathrm{oGK} / \mathrm{pW}, \mathrm{oGK} / \mathrm{pGK}$ and $\mathrm{oW} / \mathrm{gW}$ groups. Mean body weight was significantly higher $(p<0.001)$ in the $o \mathrm{GK} / \mathrm{pW}$ fetuses than in the oGK/pGK fetuses, and became similar to that of $\mathrm{oW} / \mathrm{pW}$ fetuses, indicating that the growth retardation observed in the oGK/pGK fetuses was related to the in utero diabetic environment.

At E18.5, oGK/pW fetuses, similarly to oW $/ \mathrm{pW}$ fetuses, exhibited a lower plasma glucose concentration $(p<0.001)$ than the oGK/pGK and GK stock group. Their pancreatic weights were not significantly different from values in the $\mathrm{oW} / \mathrm{pW}$ and oGK/pGK groups. In contrast, their BCMs were still 2.7-fold decreased $(p<0.001)$ compared with values in the $\mathrm{oW} / \mathrm{pW}$ group (Fig. 1 and Table 1). oGK/pW fetuses exhibited a $71 \%$ reduction in the number of $\mathrm{SOX}^{+}$endocrine progenitors at E18.5, together with a decreased number of $\mathrm{PDX}^{+} /$ insulin $^{-}$cells and $\mathrm{NGN}^{+}$cells (respectively, by $63 \%$ and $67 \%$ ), compared with control oW/pW fetuses (Fig. 2). oGK/ $\mathrm{pW}$ fetuses showed significantly $(p<0.05)$ decreased intrapancreatic accumulation of IGF2 and IGF1R proteins (by $52 \%$ and $17 \%$, respectively) compared with control oW/pW values (Fig. 1 and Table 1). These characteristics in the oGK/ $\mathrm{pW}$ fetuses were not significantly different from those of oGK/pGK fetuses. Taken together the data from the oGK/ $\mathrm{pW}$ group suggest that the early reduction in BCM in the GK/ Par model is related to multiple defects in the embryonic development of beta cells, which are largely independent of the maternal diabetic milieu. oGK/pW ACM was not significantly different from oGK/pGK or oW/pW values (Fig. 1 and Table 1).

Adult oGK/pW/sGK offspring have a full diabetic phenotype To assess any possible remote effect of the diabetic antenatal environment (from earliest preimplantation stage to birth) on GK adult glucose homeostasis, we studied adult offspring from six litters of GK rats from GK ovocyte transfer into $\mathrm{W}$ females and suckled by GK foster mothers (oGK/ $\mathrm{pW} / \mathrm{sGK}$ ). Comparison was made with values in the oW/ $\mathrm{pW} / \mathrm{sW}$ and oGK/pGK/sGK groups. From the data obtained (Figs 3 and 4, Table 2, ESM Results), one can conclude unequivocally that the intrauterine development of a GK 

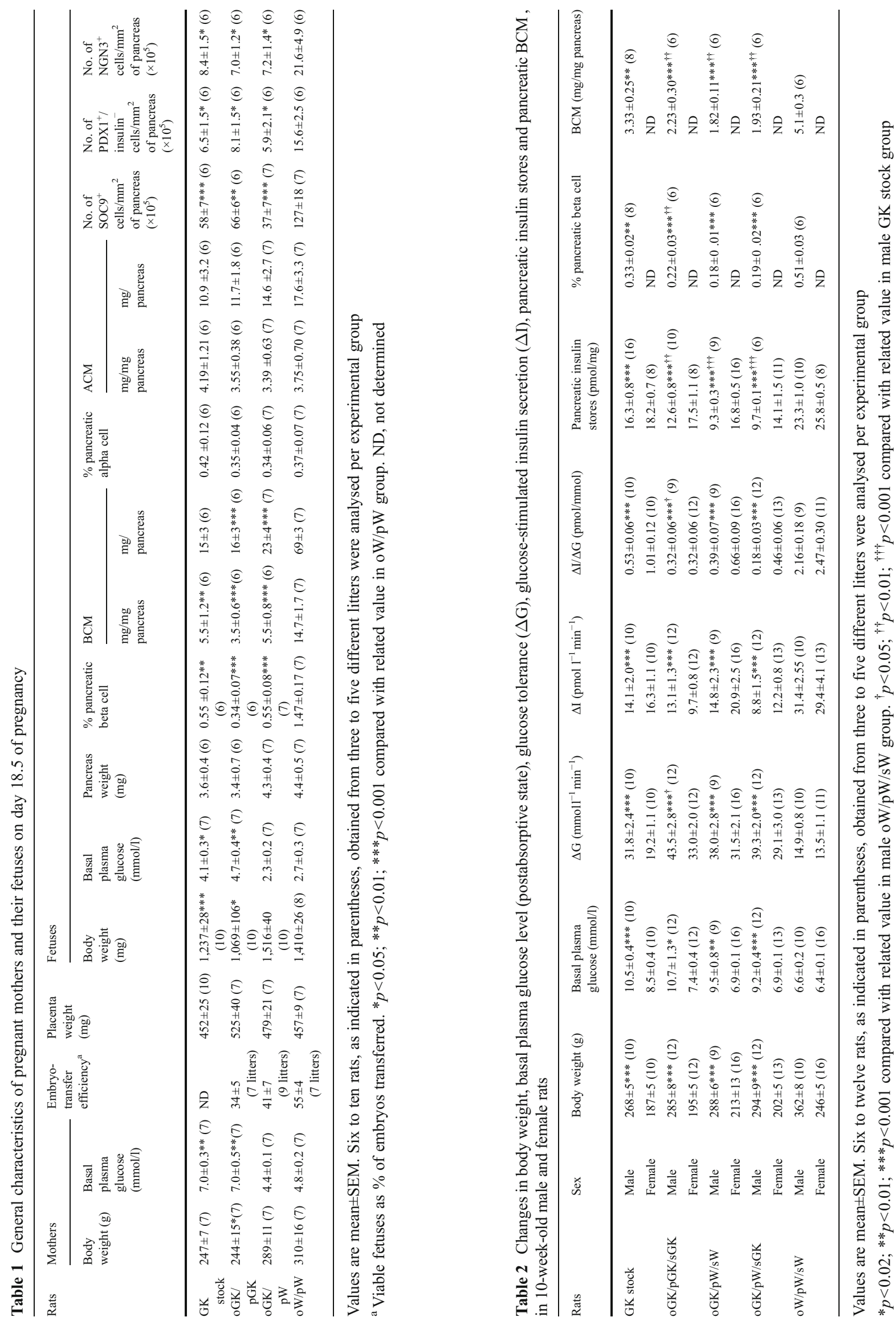

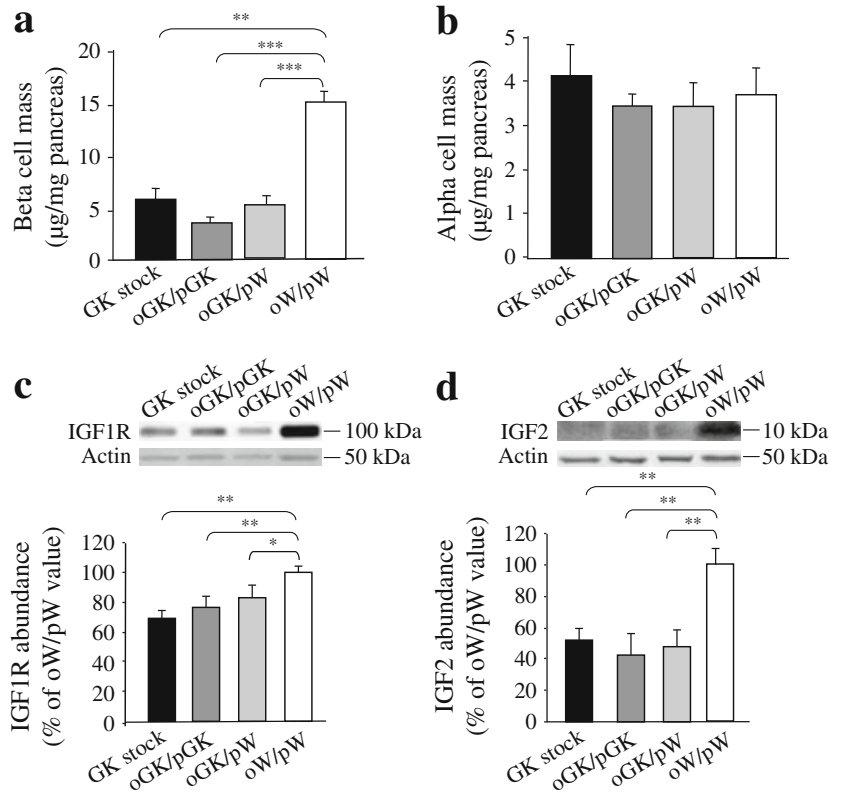

Fig. 1 Beta cell mass (a), alpha cell mass (b), IGF1R protein accumulation (c) and IGF2 protein accumulation (d) in E18.5 fetal pancreases. Representative western immunoblots in (c) and (d). Densitometric measurements $(\mathbf{c}, \mathbf{d})$ of western immunoblot bands are expressed as percentages of the corresponding oW/pW values. The fetuses were obtained from GK/Par rats (no transfer) (GK stock), GK/ Par ovocyte transfer into GK/Par mothers (oGK/pGK group), GK/Par ovocyte transfer into $\mathrm{W}$ mothers (oGK/pW group), or W ovocyte transfer into $\mathrm{W}$ mothers (oW/pW group). Values are mean $\pm \mathrm{SEM}$. Six or seven rats from three to five different litters were analysed per experimental group. ${ }^{*} p<0.05,{ }^{* *} p<0.01,{ }^{* * *} p<0.001$ compared with related value in $\mathrm{oW} / \mathrm{pW}$ group

offspring into a $\mathrm{W}$ non-diabetic mother and its subsequent breeding by a GK diabetic mother does not reduce the risk of diabetes at an adult age, regardless of the sex.

Adult oGK/pW/sWoffspring have a full diabetic phenotype To assess any possible remote effect of the diabetic perinatal environment (from earliest preimplantation stage to weaning) on GK adult glucose homeostasis, we studied adult offspring from six litters of GK rats from GK ovocyte transfer into $\mathrm{W}$ females and suckled by their $\mathrm{W}$ mothers (oGK/pW/sW). Comparison was made with values in the $\mathrm{oW} / \mathrm{pW} / \mathrm{sW}$ and $\mathrm{oGK} / \mathrm{pGK} / \mathrm{sGK}$ groups. From the data obtained (Figs 3 and 4, Table 2, ESM Results), one can conclude unequivocally that the intrauterine development of a GK offspring into a W non-diabetic mother and its subsequent breeding by a non-diabetic mother does not reduce the risk of diabetes at an adult age, regardless of the sex.

Adult phenotype of $n G K / s W$ offspring To assess any possible long-term effect of the diabetic postnatal suckling environment alone (from birth to weaning) on glucose homeostasis, we studied adult male offspring from six litters of GK newborns (nGK) from GK mothers and suckled by $\mathrm{W}$ foster mothers (nGK/sW). At 4 weeks (just before weaning) and 8 weeks of age, comparison was made with phenotypes in $\mathrm{nW} / \mathrm{sW}$ and $\mathrm{nGK} / \mathrm{sGK}$ groups raised in parallel. From the data obtained (ESM Results and ESM Table 1), we concluded that suckling of a GK offspring by a non-diabetic mother does not lower the risk of diabetes at an adult age.

\section{Discussion}

The methodology used to establish the GK rat line implies that genes predisposing to diabetes present in a non-diabetic outbred W strain were concentrated through repeated selective breeding with glucose intolerance as a selection index [10]. The search for morbid genes using a quantitative trait locus (QTL) approach has led to identification of six independently segregating-loci-containing genes involved in glucose homeostasis in GK/Par rats [12]. The same conclusion was drawn by Galli et al [19] using GK/Sto rats.
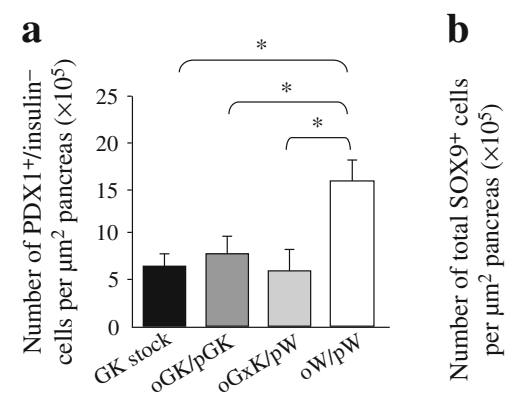
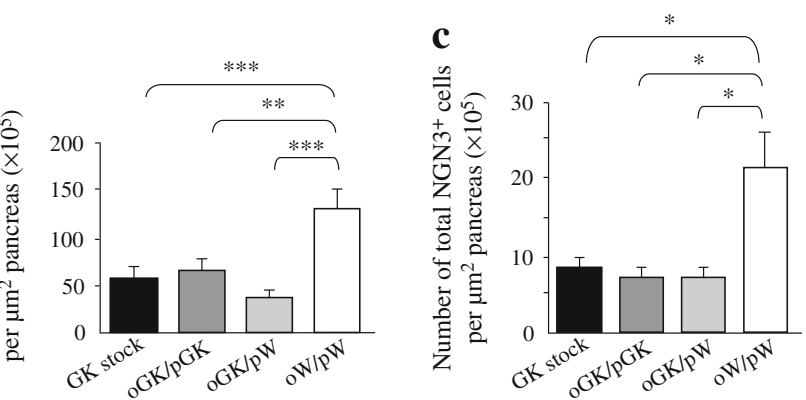

Fig. 2 Expression of progenitor cell markers in E18.5 fetal pancreases. The fetuses were obtained from GK/Par rats (no transfer) (GK stock), GK/Par ovocyte transfer into GK/Par mothers (oGK/pGK group), GK/ Par ovocyte transfer into $\mathrm{W}$ mothers (oGK/pW group), or $\mathrm{W}$ ovocyte transfer into $\mathrm{W}$ mothers (oW/pW group). (a) Sections were doublestained for PDX1 and insulin. Total PDX ${ }^{+} /$insulin ${ }^{-}$cells were counted and results expressed as number per $\mu \mathrm{m}^{2}$ section area. (b) Sections

were stained for SOX9. Total $\mathrm{SOX}^{+}$cells were counted and results expressed as number per $\mu \mathrm{m}^{2}$ section area. (c) Sections were stained for $\mathrm{NGN} 3$. $\mathrm{Ngn}^{+}$cells were calculated as the percentage of total cells. Six or seven rats obtained from three to five different litters were analysed per experimental group. ${ }^{*} p<0.05,{ }^{*} p<0.01,{ }^{* * *} p<0.001$ compared with related value in $\mathrm{oW} / \mathrm{pW}$ group 


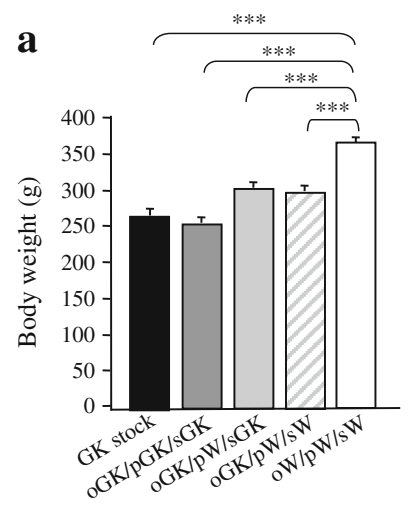

b

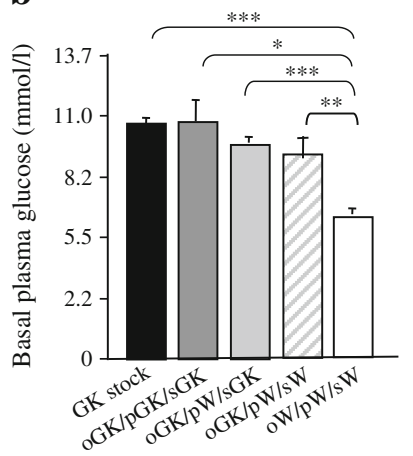

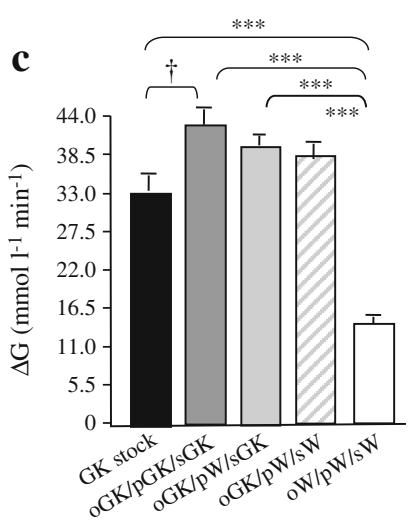

Fig. 3 Body weight (a), basal plasma glucose level (postabsorptive state) (b) and glucose tolerance $(\Delta \mathrm{G})(\mathbf{c})$ in 10-week-old male rats obtained from GK/Par ovocyte transfer into $\mathrm{W}$ mothers and reared with $\mathrm{W}$ dams (oGK/pW/sW group), in 10-week-old male rats obtained from GK/Par ovocyte transfer into W mothers and reared with GK/Par dams (oGK/pW/sGK group), in 10-week-old male rats obtained from GK/ Par ovocyte transfer into GK/Par mothers and reared with GK/Par dams (oGK/pGK/sGK group), in 10-week-old male control (no

Besides the role of GK susceptibility loci, the possibility exists that the GK maternal diabetic environment per se causes early changes in the structure and function of several organs in the offspring, including the endocrine pancreas, and has profound influence on glucose handling by the fetus, which persists into adult life and the subsequent generation of offspring.

The first strategy used to evaluate potential maternal influence on the development of diabetes was to intercross GK and W rats [19-21]. One study has reported that offspring of GK male/W female crosses are less hyperglycaemic than those of GK female/W male crosses, and this was taken as evidence of the diabetogenic effect of exposure transfer) GK/Par rats (GK stock), or in 10-week-old male rats obtained from $\mathrm{W}$ ovocyte transfer into $\mathrm{W}$ mothers and reared with $\mathrm{W}$ dams (oW/ $\mathrm{pW} / \mathrm{sW}$ group). In vivo glucose tolerance was tested with an i.v. injection of glucose $(0.5 \mathrm{~g} / \mathrm{kg}$ body weight $)$ in $5 \mathrm{~h}$-fasted anaesthetised rats. Values are mean $\pm \mathrm{SEM}$. Nine to 12 rats were analysed per experimental group. ${ }^{*} p<0.02,{ }^{*} p<0.01,{ }^{* * *} p<0.001$ compared with related value in male oW/pW/sW group; ${ }^{\dagger} p<0.05$ compared with related value in male GK stock group

to maternal diabetes in utero [20]. These maternal effects have not been confirmed in other studies [21-23]. In fact, all these experiments are hampered by the fact that the offspring share parental non-diabetogenic and diabetogenic genes. An ideal design for testing the isolated impact of diabetic GK pregnancy on the GK embryo would allow the GK embryo to become exposed to normoglycaemia throughout pregnancy only. It has been proposed that the strategy of GK embryo transfer to a W mother offers such an opportunity [9].

Our study focused first on early development of BCM in the oGK/pW fetuses. Our E18.5 results show that, despite a normal non-diabetic environment in utero, $\mathrm{BCM}$ in the a

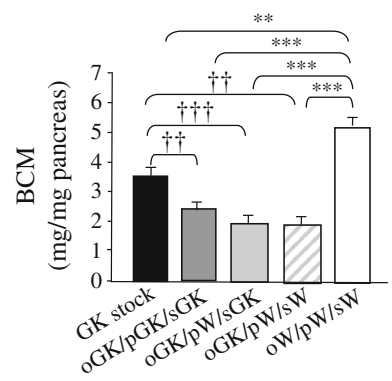

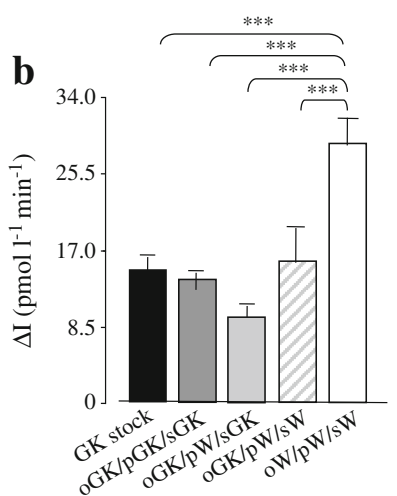

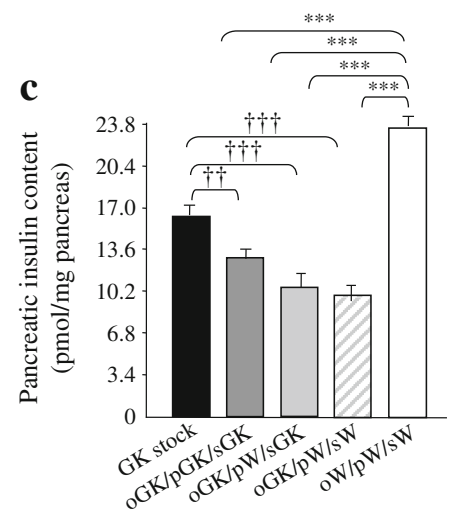

Fig. 4 BCM (a), glucose-stimulated insulin secretion $(\Delta \mathrm{I})(\mathbf{b})$ and pancreatic insulin stores (c) in 10-week-old male and female rats obtained from GK/Par ovocyte transfer into $\mathrm{W}$ mothers and reared with $\mathrm{W}$ dams (oGK/pW/sW group), in 10-week-old rats obtained from GK/Par ovocyte transfer into W mothers and reared with GK/Par dams (oGK/pW/sGK group), in 10-week-old rats obtained from GK/Par ovocyte transfer into GK/Par mothers and reared with GK/Par dams (oGK/pGK/sGK group), in 10-week-old control (no transfer) GK/Par rats (GK stock), or in 10-week-old rats obtained from $\mathrm{W}$ ovocyte transfer into $\mathrm{W}$ mothers and reared with $\mathrm{W}$ dams (oW/pW/sW group). $\Delta \mathrm{I}$ was calculated from values obtained during the glucose tolerance tests. Values are mean \pm SEM. Six to 12 rats were analysed per experimental group. $* * p<0.01, * * * p<0.001$ compared with related value in male oW/pW/sW group; ${ }^{\dagger \dagger} p<0.01,{ }^{\dagger \dagger \dagger} p<0.001$ compared with related value in male GK stock group 
$\mathrm{oGK} / \mathrm{pW}$ fetuses was still impaired whereas ACM remained unaltered. It should be noted that this happened despite a return to normal fetal growth and pancreas weight, clearly illustrating a lack of association between fetal weight and development of BCM (and further risk of type 2 diabetes). Our findings in the oGK/pW fetus do not necessarily contradict the evidence for an adverse influence of maternal diabetes on beta cell formation, as reported in the majority of experimental studies with maternal diabetes induced (not spontaneous) in various models [24]. Beta cell development, differentiation and survival are indisputably controlled by genes. Expression of these genes can be altered by mutations and/or epigenetic modifications orchestrated by the cell environment. For example, glucose is now recognised to be an important modulator of pancreatic endocrine cell differentiation through its control of production of the transcription factors, NGN3 and NeuroD [25], and hyperglycaemia correlates with DNA hypomethylation and aberrant gene expression in a diabetic zebrafish model [26]. Some of these genes (Igf2, Igflr, Pdx1, Sox9, Neurog3) are not correctly expressed in fetal GK pancreatic tissue (this study). The reason is not known. One can imagine that some of these genes are imprinted by the maternal diabetic environment (epigenetic modifications). Alternatively, but not exclusively, one can also imagine that some genes are point mutated and have been selected during the obtention of the GK line. Note that Igf2 belongs to one of the six diabetic loci of the GK rat [12], and a QTL linked to BCM was recently reported in $\mathrm{GK} / \mathrm{Ox}$ rats [27]. Our data may also be interpreted as the inability of the maternal diabetes environment to affect strong genetic factors involved in the alteration of the GK BCM development. The 10-week-old adult offspring of the oGK/pW/sGK group (oGK transferred into $\mathrm{W}$ uterus and fed by GK foster mothers) had a $60 \%$ reduction in their pancreatic insulin stores and BCM, compared with the oW/pW/sW group, to an extent even more severe than that of the oGK/pGK/sGK group. They exhibited a diabetic phenotype (body weight, basal plasma glucose and insulin, glucose tolerance and in vivo glucose-induced insulin secretion) as severe as that of the oGK/pGK/sGK rats and the GK stock rats. This was true regardless of sex. Thus normalisation of the perinatal environment in the GK/ Par mother does not remove early BCM alteration and subsequent diabetes risk in the GK model.

Early postnatal life is also important for the development and later function of beta cells, and it is clear that altered growth profiles in the postnatal period can affect adult metabolic homeostasis [28-30]. This is a matter of concern in the GK model, since we observed growth retardation during neonatal life in GK pups reared by GK mothers (nGK/sGK group) compared with $\mathrm{W}$ reared by non-diabetic $\mathrm{W}$ mothers (nW/sW group). Increased glucose levels as well as decreased milk synthesis and milk ejection have been reported in lactating streptozotocin-induced diabetic rats [31]. If this also applies to GK/Par mothers, it may lead to both qualitative and quantitative alterations in nutrition, culminating in undernutrition of the $\mathrm{nGK} / \mathrm{sGK}$ pups. Moreover, other factors, such as the maternal behaviour that is altered in the GK/Par model (D. Bailbé, J. Movassat and B. Portha, unpublished observation), may contribute to the outcome. However, the postnatal growth retardation observed in the $\mathrm{nGK} / \mathrm{sGK}$ offspring was not improved in GK neonates suckled by $\mathrm{W}$ foster mothers (nGK/sW group). Comparison of $n G K / s G K$ and $n G K / s W$ phenotypes suggests that a postnatal diabetic environment has no significant effect on BCM programming, since BCM and pancreatic insulin stores were decreased to the same extent in both groups. Also, at an adult age (10 weeks), the diabetic phenotypes were similar in the $\mathrm{nGK} / \mathrm{sGK}$ and $\mathrm{nGK} /$ $\mathrm{sW}$ groups, and also similar to that in the adult oGK $/ \mathrm{gW} / \mathrm{sW}$ group. These convergent data support our interpretation that the early BCM alteration and subsequent diabetes risk in the GK model is independent of the immediate postnatal (suckling) environment.

Our interpretation of the present observations in the oGK/ $\mathrm{pW} / \mathrm{sGK}$ and oGK/pW/sW offspring is that, despite a normal intrauterine and/or postnatal nutritional/metabolic environment being provided, the inherited diabetic pattern is not removed. The notion that inheritance of morbid genes plays a prominent role in the defective development of BCM in GK rats is also in accordance with previous studies from our group. Offspring from crosses between GK/Par and W rats showed that F1 hybrid fetuses, regardless of whether the mother was a GK or W rat, exhibited decreased BCM and glucose-stimulated insulin secretion, closely resembling GK/ GK fetuses [32]. We have also shown that, to have one GK parent is a risk factor for a low-BCM phenotype in young adults, even when the other parent is a normal $\mathrm{W}$ rat [21].

In conclusion, the present study supplies strong evidence that the low-BCM phenotype and its associated diabetes risk in adult GK/Par rats is not removed by normalisation of the prenatal and/or postnatal diabetic environments. Importantly, the lack of impact on offspring with the GK genotype once maternal diabetes has been eliminated does not mean that maternal diabetes has no effect on offspring with a normal genotype (W rat): we have preliminary data based on W ovocyte transfer to diabetic GK/Par females suggesting that GK maternal diabetes negatively imprints growth of the genetically normal W BCM. Therefore our data do not rule out any influence of epigenetic mechanisms induced by maternal diabetes. We are, however, left with no clear picture about the nature and role of diabetes-predisposing genes, especially those involved in defective BCM, which is certainly the primary functional defect involved in the pathogenesis of diabetes in the GK rat. Previous investigations in the GK model using therapeutic agents such as glucagon-like peptide 1 receptor agonists [33] during early 
developmental stages of a given generation showed efficient amelioration of the adult phenotype (improved BCM and milder diabetes). Whether correction of pancreatic deficiencies in a given ' $n$ ' generation is likely to overcome the heritable BCM dysregulation in the ' $n+1$ ' generation remains an open question.

Acknowledgements Some of these data were presented at the 68th American Diabetes Association Meeting, San Francisco, USA, 18-21 June 2008 and at the 44th European Association for the Study of Diabetes Meeting, Roma, Italy, 8-11 September 2008.

Funding These studies were funded by the French ANR (programme Physio 2006 - Prograbeta - ref ANR-06-PHYSIO-028) and the NEB Association. A. Chavey received a CNRS postdoctoral fellowship, granted by NESTLE-France and Alfediam/SFD.

Duality of interest The authors declare that there is no duality of interest associated with this manuscript.

Contribution statement AC, DB and LM contributed to the experiments and participated in the analysis and interpretation of the data. JPR, JM and BP participated in the analysis and interpretation of the data. JPR and BP conceived and designed the study and obtained funding. BP drafted the manuscript, and all other authors revised it critically for intellectual content. All authors approved the final version of the paper.

\section{References}

1. Dorner G, Mohnike A (1976) Further evidence for a predominantly maternal transmission of maturity-onset type diabetes. Endokrinologie $68: 121-124$

2. Martin AO, Simpson JL, Ober C, Freinkel N (1985) Frequency of diabetes mellitus in mothers of probands with gestational diabetes: possible maternal influence on the predisposition to gestational diabetes. Am J Obstet Gynecol 151:471-475

3. Harder T, Franke K, Kohlhoff R, Plagemann A (2001) Maternal and paternal family history of diabetes in women with gestational diabetes or insulin-dependent diabetes mellitus type I. Gynecol Obstet Invest 51:160-164

4. McCarthy M, Cassell P, Tran T et al (1996) Evaluation of the importance of maternal history of diabetes and of mitochondrial variation in the development of NIDDM. Diabet Med 13:420 428

5. Mitchell BD, Valdez R, Hazuda HP, Haffner SM, Monterrosa A, Stern MP (1993) Differences in the prevalence of diabetes and impaired glucose tolerance according to maternal or paternal history of diabetes. Diabetes Care 16:1262-1267

6. Kim DJ, Cho NH, Noh JH, Lee MS, Lee MK, Kim KW (2004) Lack of excess maternal transmission of type 2 diabetes in a Korean population. Diabetes Res Clin Pract 65:117-124

7. Knowler WC, Bennett PH, Hamman RF, Miller M (1978) Diabetes incidence and prevalence in Pima Indians: a 19-fold greater incidence than in Rochester, Minnesota. Am J Epidemiol 108:497505

8. Sobngwi E, Boudou P, Mauvais-Jarvis F et al (2003) Effect of a diabetic environment in utero on predisposition to type 2 diabetes. Lancet 361:1861-1865
9. Gill-Randall R, Adams D, Ollerton RL, Lewis M, Alcolado JC (2004) Type 2 diabetes mellitus: genes or intrauterine environment? An embryo transfer paradigm in rats. Diabetologia 47:1354-1359

10. Goto Y, Kakizaki M, Musaki N (1976) Production of spontaneous diabetic rats by repetition of selective breeding. Tohoku J Exp Med 119:85-90

11. Portha B, Lacraz G, Chavey A et al (2010) Islet structure and function in the GK rat. Adv Exp Med Biol 654:479-500

12. Gauguier D, Froguel P, Parent V et al (1996) Chromosomal mapping of genetic loci associated with non-insulin dependent diabetes in the GK rat. Nat Genet 12:38-43

13. Cozzi J, Wang E, Jacquet C, Fraichard A, Cherifi Y, Zhou Q (2010) Procedures for somatic cell nuclear transfer in the rat. Methods Mol Biol 597:137-150

14. Movassat J, Saulnier C, Serradas P, Portha B (1997) Impaired development of pancreatic beta-cell mass is a primary event during the progression to diabetes in the GK rat. Diabetologia 40:916-925

15. Figeac F, Uzan B, Faro M, Chelali N, Portha B, Movassat J (2010) Neonatal growth and regeneration of beta-cells are regulated by the Wnt/beta-catenin signaling in normal and diabetic rats. Am J Physiol Endocrinol Metab 298:E245-E256

16. Caldérari S, Gangnerau MN, Thibault M et al (2007) Defective IGF-2 and IGF-1R protein expressions in embryonic pancreas precede beta-cell mass anomaly in GK rat. Diabetologia 50:14631471

17. Lynn FC, Smith SB, Wilson ME, Yang KY, Nekrep N, German MS (2007) Sox9 coordinates a transcriptional network in pancreatic progenitor cells. Proc Natl Acad Sci USA 104:10500-10505

18. Serradas P, Goya L, Lacorne M, Gangnerau MN, Ramos S, Alvarez C, Pascual-Leone AM, Portha B (2002) Fetal insulinlike growth factor-2 production is impaired in the GK rat model of type 2 diabetes. Diabetes 51:392-397

19. Galli J, Li LS, Glaser A et al (1996) Genetic analysis of noninsulin dependent diabetes mellitus in the GK rat. Nat Genet 12:31-37

20. Gauguier D, Nelson I, Barnard C et al (1994) Higher maternal than paternal inheritance of diabetes in GK rats. Diabetes 43:220 224

21. Calderari S, Gangnerau MN, Meile MJ, Portha B, Serradas P (2006) Is defective pancreatic beta-cell mass environmentally programmed in Goto Kakizaki rat model of type-2 diabetes? Insights from cross-breeding studies during the suckling period. Pancreas 33:412-417

22. Abdel-Halim SM, Guenifi A, Luthman H, Grill V, Efendic S, Ostenson CG (1994) Impact of diabetic inheritance on glucose tolerance and insulin secretion in spontaneously diabetic GKWistar rats. Diabetes 43:281-288

23. Gill-Randall RJ, Adams D, Ollerton RL, Alcolado JC (2004) Is human type 2 diabetes maternally inherited? Insights from an animal model. Diabet Med 21:759-762

24. Jawerbaum A, White V (2010) Animal models in diabetes and pregnancy. Endocr Rev 31:680-701

25. Guillemain G, Filhoulaud G, Da Silva-Xavier G, Rutter GA, Scharfmann R (2007) Glucose is necessary for embryonic pancreatic endocrine cell differentiation. J Biol Chem 282:15228-15237

26. Olsen AS, Sarras MP Jr, Leontovich A, Intine RV (2012) Heritable transmission of diabetic metabolic memory in zebrafish correlates with DNA hypomethylation and aberrant gene expression. Diabetes 61:485-491

27. Finlay C, Argoud K, Wilder SP et al (2010) Chromosomal mapping of pancreatic islet morphological features and regulatory hormones in the spontaneously diabetic (type 2) Goto-Kakizaki rat. Mamm Genome 21:499-508

28. Knittle JL, Hirsch J (1968) Effect of early nutrition on the development of rat epididymal fat pads: cellularity and metabolism. J Clin Invest 47:2091-2098 
29. Lemonnier D, Suquet JP, Aubert R, Rosselin G (1973) Long term effect of mouse neonate food intake on adult body composition, insulin and glucose serum levels. Horm Metab Res 5:223-224

30. Siebel AL, Mibus A, de Blasio MJ et al (2008) Improved lactational nutrition and postnatal growth ameliorates impairment of glucose tolerance by uteroplacental insufficiency in male rat offspring. Endocrinology 149:3067-3076

31. Lau C, Sullivan MK, Hazelwood RL (1993) Effects of diabetes mellitus on lactation in the rat. Proc Soc Exp Biol Med 204:81-89
32. Serradas P, Gangnerau MN, Giroix MH, Saulnier C, Portha B (1998) Impaired pancreatic beta cell function in the fetal GK rat. Impact of diabetic inheritance. J Clin Invest 101:899904

33. Tourrel C, Bailbé D, Lacorne M, Meile MJ, Kergoat M, Portha B (2002) Persistent improvement of type 2 diabetes in the GotoKakizaki rat model by expansion of the beta-cell mass during the prediabetic period with Glucagon-like peptide-1 or Exendin-4. Diabetes 51:1443-1452 